To appear in ApJ

\title{
Constraining the Physical Properties of Near-Earth Object 2009 BD
}

\author{
M. Mommert \\ Department of Physics and Astronomy, Northern Arizona University, PO Box 6010, \\ Flagstaff, AZ 86011, USA \\ michael.mommert@nau.edu \\ J. L. Hora \\ Harvard-Smithsonian Center for Astrophysics, 60 Garden Street, MS 65, Cambridge, MA \\ 02138-1516, USA \\ D. Farnocchia \\ Jet Propulsion Laboratory, California Institute of Technology, Pasadena, CA 91109, USA \\ S. R. Chesley \\ Jet Propulsion Laboratory, California Institute of Technology, Pasadena, CA 91109, USA \\ D. Vokrouhlický \\ Institute of Astronomy, Charles University, V Holešovičkách 2, CZ-18000, Prague 8, Czech \\ Republic \\ D. E. Trilling \\ Department of Physics and Astronomy, Northern Arizona University, PO Box 6010, \\ Flagstaff, AZ 86011, USA \\ M. Mueller \\ SRON Netherlands Institute for Space Research, Postbus 800, 9700 AV, Groningen, The \\ Netherlands \\ A. W. Harris \\ DLR Institute of Planetary Research, Rutherfordstrasse 2, 12489 Berlin, Germany
}


H. A. Smith

Harvard-Smithsonian Center for Astrophysics, 60 Garden Street, MS 65, Cambridge, MA 02138-1516, USA

and

G. G. Fazio

Harvard-Smithsonian Center for Astrophysics, 60 Garden Street, MS 65, Cambridge, MA 02138-1516, USA

\begin{abstract}
We report on Spitzer Space Telescope IRAC observations of near-Earth object (NEO) 2009 BD that were carried out in support of the NASA Asteroid Robotic Retrieval Mission (ARRM) concept. We did not detect 2009 BD in 25 hrs of integration at $4.5 \mu \mathrm{m}$. Based on an upper-limit flux density determination from our data, we present a probabilistic derivation of the physical properties of this object. The analysis is based on the combination of a thermophysical model with an orbital model accounting for the non-gravitational forces acting upon the body. We find two physically possible solutions. The first solution shows $2009 \mathrm{BD}$ as a $2.9 \pm 0.3 \mathrm{~m}$ diameter rocky body $\left(\rho=2.9 \pm 0.5 \mathrm{~g} \mathrm{~cm}^{-3}\right)$ with an extremely high albedo of $0.85_{-0.10}^{+0.20}$ that is covered with regolith-like material, causing it to exhibit a low thermal inertia $\left(\Gamma=30_{-10}^{+20}\right.$ SI units). The second solution suggests $2009 \mathrm{BD}$ to be a $4 \pm 1 \mathrm{~m}$ diameter asteroid with $p_{V}=0.45_{-0.15}^{+0.35}$ that consists of a collection of individual bare rock slabs $(\Gamma=2000 \pm 1000$ SI units,

$\left.\rho=1.7_{-0.4}^{+0.7} \mathrm{~g} \mathrm{~cm}^{-3}\right)$. We are unable to rule out either solution based on physical reasoning. $2009 \mathrm{BD}$ is the smallest asteroid for which physical properties have been constrained, in this case using an indirect method and based on a detection limit, providing unique information on the physical properties of objects in the size range smaller than $10 \mathrm{~m}$.
\end{abstract}

Subject headings: minor planets, asteroids: individual (2009 BD) - infrared: planetary systems

\title{
1. Introduction
}

The physical properties of near-Earth objects (NEOs) provide important hints on their origin, as well as their past physical and orbital evolution. The most accessible physical 
properties are the diameter, $d$, and the geometric albedo, $p_{V}$, which have been measured for more than 1000 NEOs with diameters down to slightly less than $100 \mathrm{~m}$ in two large-scale programs, the Warm Spitzer NEO survey "ExploreNEOs" (Trilling et al. 2010), and the "NEOWISE" project (Mainzer et al. 2011), using the Wide-field Infrared Survey Explorer (WISE, Wright et al. 2010). Recently, Mainzer et al. (2014) measured the sizes and albedos of the smallest optically discovered NEOs $(d>10 \mathrm{~m})$ from NEOWISE data. Little is known about the physical properties of even smaller NEOs, which constitute the bulk of the NEO population. Knowledge of the physical properties of such small NEOs, some of which pose an impact threat to the Earth, is of importance for understanding their evolution and estimating the potential of destruction in case of an impact, as well as for designing the most promising mitigation mission.

Further information on asteroid physical properties are available only for select objects with relatively large sizes, which make up only a fraction of the whole asteroid population. Such properties include, but are not limited to, the bulk density, $\rho$, thermal inertia, $\Gamma$, and the obliquity, $\gamma$, all of which affect non-gravitational forces that act upon the body and alter its orbit compared to a Keplerian one. Two important effects are the Yarkovsky effect (see, e.g., Bottke et al. 2006) and the solar radiation pressure (Vokrouhlický \& Milani 2000).

The bulk density, $\rho$, provides the simplest way of gaining insight into asteroid interiors. Solid rock, or monolithic, bodies have high bulk densities $\left(\rho \sim 3 \mathrm{~g} \mathrm{~cm}^{-3}\right)$, whereas those of rubble-pile bodies, aggregates of smaller particles that are consolidated by their selfgravity or other adhesive forces (Chapman 1978), can be significantly lower as a result of "macroporosity." Macroporosity refers to cavities and void spaces that occur between the irregularly shaped individual constituents (see Richardson et al. 2002; Britt et al. 2002, for a discussion). Britt et al. (2002) found that most asteroids show a significant degree of macroporosity, in support of the hypothesis that most asteroids must have been disrupted in the course of high-velocity impacts over the age of the Solar System (Chapman 1978). Small asteroids are generally thought of as being individual pieces of compact debris that were generated in disruptive collisions (Pravec et al. 2002); hence, their macroporosity is expected to be low and their bulk density high compared to that of rubble pile asteroids.

Thermal inertia, $\Gamma$, describes the ability of the surface material to store thermal energy: high-thermal-inertia material heats up slowly and re-emits the thermal energy only gradually, whereas low-thermal-inertia material can be approximated as being in instantaneous thermal equilibrium with the incoming insolation (see, e.g., Spencer et al. 1989). Examples for materials of low and high thermal inertia are regolith (30-50 SI units, Spencer et al. 1989; Putzig et al. 2005, 1 SI unit equals $1 \mathrm{~J} \mathrm{~m}^{-2} \mathrm{~s}^{-0.5} \mathrm{~K}^{-1}$ ) and bare rock (>2500 SI units, Jakosky 1986), respectively. Measurements of the thermal inertia of medium-to-large sized 
NEOs $(d>100 \mathrm{~m})$ revealed values of 100-1000 SI units (Delbo' et al. 2007).

Both the thermal inertia and the bulk density of asteroids can be derived by modeling the effect of non-gravitational perturbations on the object's orbit (see, e.g., Chesley et al. 2014). Assuming a homogeneous bulk density of the constituent particles, usually derived from laboratory measurements of meteorite equivalent material, allows for constraining the degree of macroporosity of the asteroid.

NEO 2009 BD was discovered on January 16, 2009, at a distance from the Earth of only 0.008 au (Buzzi et al. 2009). Its orbit is very Earth-like with a period of 400 days (JPL Solution 41). The escape velocity of 2009 BD with respect to the Earth is among the lowest for known objects $\left(v_{\infty} \sim 1 \mathrm{~km} \mathrm{~s}^{-1}\right)$, making it a worthwhile candidate mission target.

2009 BD is considered the primary candidate mission target for NASA's Asteroid Robotic Retrieval Mission (ARRM, NASA Asteroid Initiative Website 2013). The mission concept involves capturing an asteroid and dragging it onto a new trajectory that traps it in the Earth-Moon system, where it will be further investigated by astronauts. As a result of 2009 BD's Earth-like orbit, its next encounter with the Earth-Moon system will be in late 2022, when the proposed capture through ARRM would take place. The current mission design requires the target asteroid to have a diameter of $7-10 \mathrm{~m}$ and a total mass of 500 metric tons (NASA Solar System Exploration Mission Website 2013). The orbital parameters and absolute magnitude, $H$, which is the apparent magnitude of an object at a distance of 1 au to the Sun and the observer, of 2009 BD are well-known, providing accurate orbital predictions (Micheli et al. 2012). However, there is no albedo-independent determination of its diameter, which is a crucial variable in the ARRM mission planning.

We report here in observations of 2009 BD using the IRAC camera on the Spitzer Space Telescope, which provides the only practical means to constrain the physical properties of 2009 BD in the next decade. The main goals of our observations were two-fold: measure the size and therefore determine the suitability of 2009 BD as an ARRM mission target, and constrain other physical properties like bulk density and thermal inertia of an asteroid at a size range that is so far unprecedented.

\section{Observations}

We observed 2009 BD with the Infrared Array Camera (IRAC, Fazio et al. 2004) onboard the Spitzer Space Telescope (Werner et al. 2004) in Program ID 90256 using Director's Discretionary Time. A total of 25 hrs of observation time was split into three Astronomical Observation Requests (AORs): 49092096 (observation mid-time: Oct 13, 2013, 16:23 UTC; 
8 hrs elapsed time), 49091840 (Oct 14, 00:20 UTC; 8 hrs), and 49091584 (Oct 14, 20:54 UTC; $9 \mathrm{hrs}$ ). The observation window was selected based on Spitzer observability. Based on flux density predictions derived with the near-Earth asteroid thermal model (NEATM, Harris 1998), a detection in IRAC channel $1(3.6 \mu \mathrm{m})$ seemed to be unlikely. Hence, all available observing time was used on channel $2(4.5 \mu \mathrm{m})$ observations, where the predicted flux density was greater than the predicted $5 \sigma$ IRAC channel 2 sensitivity during the observation window.

In our observations, individual AORs used the "Moving Single" object mode to track in the moving frame of 2009 BD. A medium cycling dither pattern was used with a $100 \mathrm{sec}$ frame time. In order to provide the most accurate pointing during our observations, the JPL Horizons online Solar System data and ephemeris computation routine, which provides the Spitzer pointing information, was updated to include non-gravitational effects in the prediction of the orbit. We modeled non-gravitational perturbations as

$$
\mathbf{a}_{N G}=\left(A_{1} \hat{\mathbf{r}}+A_{2} \hat{\mathbf{t}}\right)\left(\frac{1 \mathrm{au}}{r}\right)^{2},
$$

where $\hat{\mathbf{r}}$ and $\hat{\mathbf{t}}$ are the radial and transverse directions, respectively, and $r$ is the heliocentric distance. $A_{2} / r^{2}$ models the transverse component of the Yarkovsky effect (Bottke et al. 2006), whereas $A_{1} / r^{2}$ models the solar radiation pressure (Vokrouhlický \& Milani 2000) and the radial component of the Yarkovsky effect. This is similar to the comet-like model for non-gravitational perturbations (Marsden et al. 1973). The orbital fit (JPL Solution 41) to the observations yields $A_{1}=(57.03 \pm 7.79) \times 10^{-12} \mathrm{au} / \mathrm{d}^{2}$ and $A_{2}=(-113.02 \pm 7.89) \times 10^{-14}$ $\mathrm{au} / \mathrm{d}^{2}$ (see also the entry for 2009 BD in the JPL Small-Body Database Browser 2013, as of October 24, 2013). The correlation coefficient between $A_{1}$ and $A_{2}$ is 0.81 . The orbital fit is based on 180 optical observations over the interval from 2009-Jan-16.3 to 2011-Jun-21.0. The positional uncertainty of $2009 \mathrm{BD}$ as seen by Spitzer at the time of the observations was $\pm 5^{\prime \prime} 0$ in right ascension and \pm 0 " 4 in declination at a $3 \sigma$ confidence level. For comparison, IRAC offers a square field of view with a width of 5.2 and a pixel scale of 1 1.2/pixel (Warm Spitzer Observer's Manual 2012). Hence, the accuracy of the orbit determination for 2009 BD is sufficient to determine its position to within a few IRAC pixels (see Section 5.1 for a more detailed discussion).

The data were reduced using a method tailored to faint NEOs, based on the ExploreNEOs program (Trilling et al. 2010). In this method, a mosaic of the field is constructed from the dataset itself and then subtracted from the individual Basic Calibrated Data (BCD) frames. During these observations, the target had an apparent motion of $\sim 0{ }^{\prime \prime} 4$ during each $100 \mathrm{sec}$ frame, so background stars were trailed only very slightly in individual BCDs. We were therefore able to generate a high signal-to-noise mosaic of the field to subtract from the BCDs. After subtraction of the background mosaic, regions near the peaks of background 
sources (which had small residuals) and bright cosmic ray artifacts were also masked in the individual BCDs, in order to minimize the background noise. The processed BCDs were then mosaicked in the reference frame of the moving object for each AOR, and the results from the three AORs combined to produce a final mosaic that included the full set of 800 100-second frames.

We did not detect 2009 BD in this final co-added map (Figure 1, right), from which we derive a $3 \sigma$ upper limit to the flux density of $2009 \mathrm{BD}$ of $0.78 \mu \mathrm{Jy}$.

\section{Modeling Method}

The lack of a clear detection of 2009 BD in our observations precludes a direct determination of its physical properties. In order to be able to indirectly constrain the physical properties of $2009 \mathrm{BD}$, we take a probabilistic approach that combines a thermophysical model with a model of the non-gravitational effects on the asteroid's orbit. Based on the upper-limit flux density provided by our Spitzer observation and available astrometric measurements, the combination of both models allows us to constrain the physical properties of 2009 BD. The combination of the two models provides proper accounting for the mutual dependencies of the individual physical properties that impact both models, which would not be possible using a more simple thermal model.

We model the non-gravitational effects on the orbit of 2009 BD, namely the solar radiation pressure and the Yarkovsky effect, as a function of $d, \Gamma, \rho, \gamma$, and other parameters in

a numerical approach. For the solar radiation pressure we assume (Vokrouhlický \& Milani 2000)

$$
\mathbf{a}_{\mathrm{SRP}}=\left(1+\frac{4}{9} A\right) \cdot \Psi \cdot \frac{G_{S}}{c} \cdot \frac{\hat{\mathbf{r}}}{r^{2}},
$$

where $A$ is the Bond albedo, $\Psi$ is the area-to-mass ratio of the object (see, e.g., Micheli et al. 2012), $G_{S}=1370 \mathrm{~W} / \mathrm{m}^{2}$ is the solar constant, and $c$ is the speed of light. For the Yarkovsky effect, we use the model approach described by Vokrouhlický et al. (2000), which fully captures both the diurnal and the seasonal components of the Yarkovsky effect. The model asteroid is assumed to be spherical and the heat transfer is solved analytically using the linearized heat transfer equation (Vokrouhlický 1998; Vokrouhlický \& Farinella 1999). By fitting all available astrometric data of $2009 \mathrm{BD}$, the model derives $\rho$ and $\Gamma$ as a function of $\gamma$ and $d$, as well as the goodness-of-fit parameter $\chi^{2}$.

The thermophysical model approximates the surface temperature distribution of 2009 BD and is used in this work to determine the thermal-infrared emission from its surface as a function of its physical properties. The model accounts for the spin axis orientation (rep- 

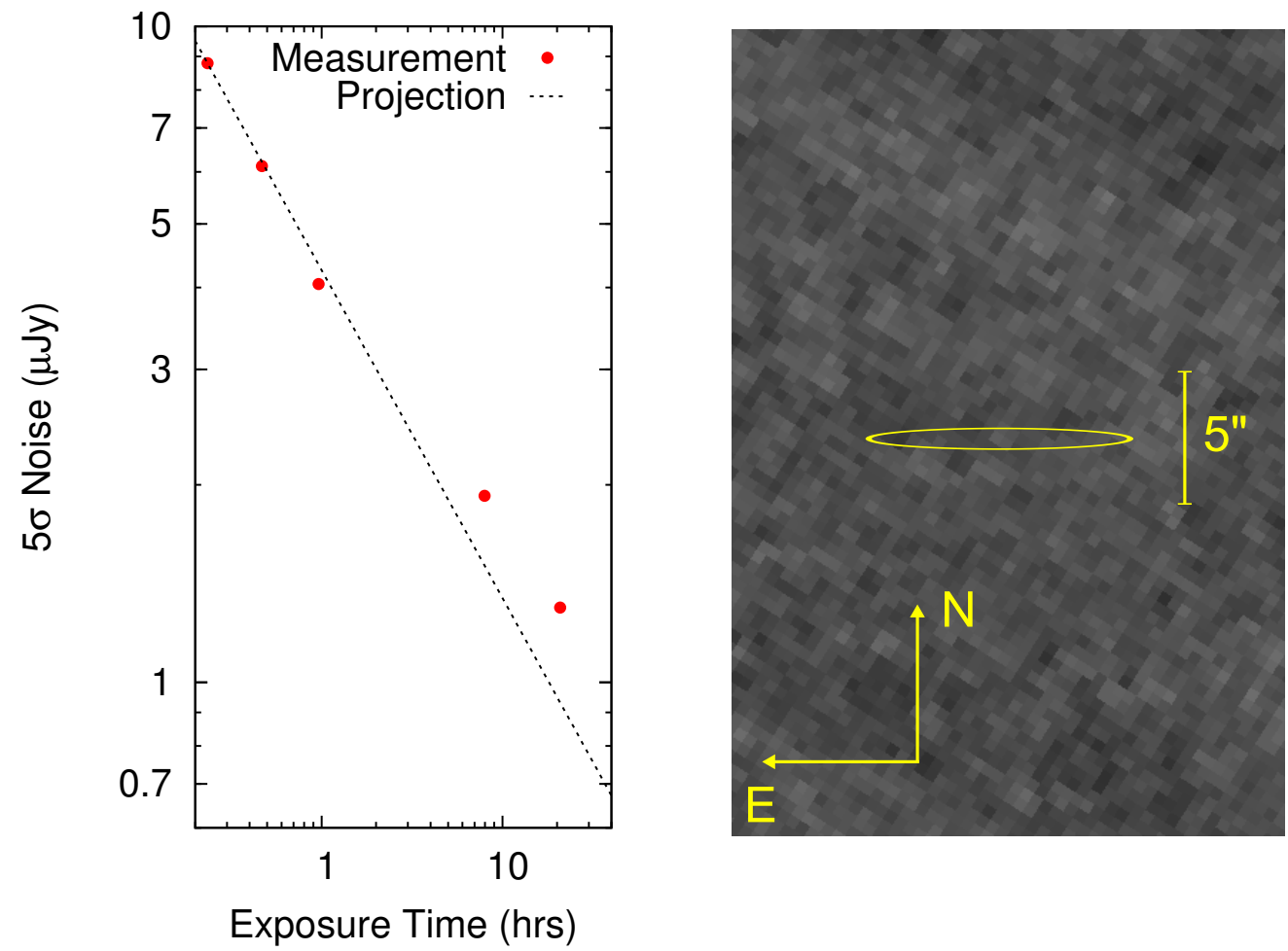

Fig. 1.- Left: IRAC channel 2 noise level prediction and measurements. Measured sensitivities are derived from ExploreNEOs data (Trilling et al. 2010, 2013). The projection is based on a $1 / \sqrt{t}$ relation, where $t$ is the integration time. The predicted sensitivity for our $25 \mathrm{hrs}$ observation was $0.78 \mu \mathrm{Jy}$ at a $5 \sigma$ level. The two data-points with the longest integration times have been measured as part of this work. Right: Excerpt from the final co-added map derived from our observations. The predicted position of 2009 BD is indicated with an ellipse, indicating the $3 \sigma$ uncertainty interval in right ascension and declination. The measured noise level is $0.78 \mu \mathrm{Jy}$ at a $3 \sigma$ level, which is higher than predicted (see left panel and the discussion in Section 5.1). 
resented by $\gamma$ ), rotational period, thermal inertia, and surface roughness. We assume a spherical shape of 2009 BD; the diameter derived with the model is hence the diameter of a sphere with the same volume as the real shape of 2009 BD. Surface roughness causes infrared beaming, an effect that focuses thermal emission radiated towards the observer, and is modeled as emission from spherical craters (see Mueller 2007, for more details). The model, which is mostly identical to the one discussed by Mueller (2007), solves the heat transfer equation numerically for a large number of plane surface facets that form a sphere. The monochromatic flux density derived by the model is turned into an IRAC channel 2 in-band flux density, i.e., it is color corrected, using the appropriate channel 2 response function and assuming a black-body spectrum of the instantaneous thermal equilibrium temperature for 2009 BD (Trilling et al. 2010). Furthermore, the contribution from reflected solar light is added to the calculated flux density using the method described by Mueller et al. (2011) (and references therein), assuming an infrared/optical reflectance ratio of 1.4.

In both the orbital and the thermophysical model we adopt the absolute magnitude $H=$ $28.43 \pm 0.12$ (Micheli et al. 2012), the photometric slope parameter $G=0.18 \pm 0.13$ (derived as the average from all $G$ measurements of asteroids, see JPL Small-Body Database Search Engine 2013), and the rotation period $P=2^{(2 \pm 0.5)}$ hrs (which is consistent with observations by Tholen et al. 2013, $P \geq 3 \mathrm{hrs}$ ) throughout this work.

\section{Results}

The mutual dependencies among physical properties used by the orbital and the thermophysical model require an iterative solution of the problem. In a first approximation, we constrain the possible ranges of $\gamma$ and $d$. As the negative value of $A_{2}$ suggests a retrograde rotation (see Farnocchia et al. 2013), we sample the obliquity $\gamma$ from $90^{\circ}$ to $180^{\circ}$. We investigate the possible range of $d$ using the thermophysical model, based on an $3 \sigma$ upper-limit flux density measurement $(0.78 \mu \mathrm{Jy})$. Figure 2 shows the predicted flux density at $4.5 \mu \mathrm{m}$ as a function of the diameter and the thermal inertia for the faintest possible model asteroid, providing the largest possible diameter range for $2009 \mathrm{BD}$ with a smooth surface, $\gamma=180^{\circ}$, and the shortest rotation period consistent with observations ( $P \geq 3 \mathrm{hrs}$, Tholen et al. 2013). From this plot, we constrain the possible diameter range of $2009 \mathrm{BD}$ to $<8 \mathrm{~m}$, as a result of our upper-limit flux density determination. Note that we do not force a lower-limit diameter constraint, so we do not apriori exclude high geometric albedos.

Sampling $d<8 \mathrm{~m}$ and $90^{\circ}<\gamma<180^{\circ}$ with the orbital model provides further constraints on 2009 BD's properties. Intriguingly, we find for each pair $(d, \gamma)$ two local minima in the orbital fit $\chi^{2}$, representing two physically possible solutions. The "low- $\Gamma$ " solution 
displays a low thermal inertia of the order of 10 SI units with a high bulk density $\rho$, whereas the "high- $\Gamma$ " solution stands out with a thermal inertia of more than 1000 SI units and a low bulk density. Figure 2 shows both solutions in thermal inertia as a function of the diameter. Based on the orbital fit solutions, we can also further constrain the obliquity (low- $\Gamma$ : $\gamma=170_{-20}^{\circ}$, high- $\Gamma: \gamma=180_{-5}^{\circ}$, uncertainties are $1 \sigma$ ) and we can confidently rule out that 2009 BD is smaller than $2.6 \mathrm{~m}$. For diameters smaller than that, the orbital model is unable to converge on a physically meaningful solution (see Section 5.2 for a detailed discussion).

We utilize our intermediate results to derive diameter distributions for both solutions using the thermophysical model, based on the thermal inertia constraints and the Spitzer upper-limit flux density measurement. We generate a sample of synthetic objects with pairs $(d, \Gamma)$ that comply with normal distributions around the thermal inertia solutions shown in Figure 2. We sample the other model input parameters $(H, G)$ according to normal distributions or log-normal distributions $(P)$ within the ranges given in Section 3 , We use $\gamma=170_{-20}^{\circ+10}$ and $\gamma=180_{-5}^{\circ+0}$ for the low- $\Gamma$ and the high- $\Gamma$ solution, respectively, and for the surface roughness we randomly pick one of four different roughness models (no, low, default, and high roughness; see Mueller 2007). We model each synthetic sample object and derive its IRAC in-band flux density combined with contributions from reflected solar light, which we then compare with the $3 \sigma$ upper-limit flux density as derived from our observations. In case the sample object flux density is lower than the upper limit, we regard this individual synthetic object a possible configuration for 2009 BD and add its diameter to the distribution. The final solution-specific diameter distributions are shown in Figure 3 and the derived nominal values and uncertainties are listed in Table 1. Nominal values represent the median values of the respective distributions; uncertainties are standard deviations, $\sigma$, of a normal distribution fitted to those values higher than the median of the distribution. Figure 3 shows that this approach reasonably describes the range of values lower than the median, which deviates from the shape of a normal distribution. We derive albedo values for the high and low- $\Gamma$ solutions similarly, using separate values of $\sigma$ for albedos higher and lower than the median, allowing for asymmetric uncertainties.

Based on the solution-specific diameter ranges, we finally constrain the other physical properties of 2009 BD using the orbital model. Figure 4shows how obliquity is constrained by the orbital fit $\chi^{2}$. By mapping the distribution in obliquity to the distributions in thermal inertia (Figure 5) and bulk density (Figure 6) we obtain our final estimates for the bulk density, thermal inertia, and total mass for both solutions, as listed in Table 1. The reported $1 \sigma$ error bars account for the uncertainties of the input physical parameters used to model the Yarkovsky accelerations (e.g., diameter and absolute magnitude) and the uncertainty resulting from the astrometry. The individual physical properties derived from both the low- $\Gamma$ and the high- $\Gamma$ solution are discussed in Section 5.2 . 


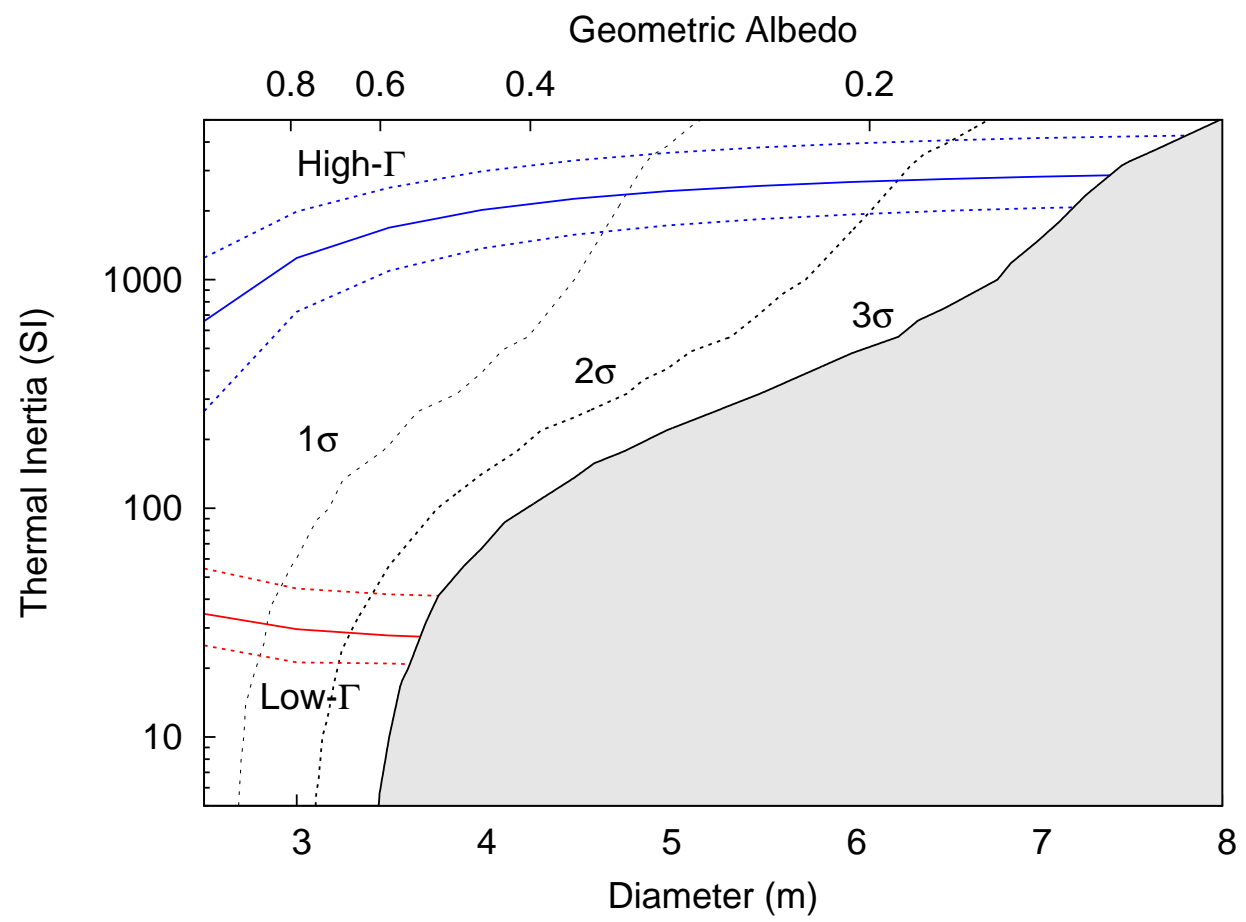

Fig. 2.- Flux density distribution of 2009 BD in diameter-thermal inertia space, according to the thermophysical model. The black [dotted, dashed, solid] lines illustrate the curvature of the area in which 2009 BD would have a flux density that is equal to our derived [ 1 , $2,3] \sigma$ upper-limit flux density. Grey areas have flux densities higher than our derived $3 \sigma$ upper-limit flux density; there is a $99.7 \%$ probability that 2009 BD must be located to the left of the black solid line. This plot is based on the assumption that 2009 BD has a smooth surface, spins rapidly $(P=3 \mathrm{~h})$, and has $\gamma=180^{\circ}$; this configuration provides the lowest possible flux densities, and hence the largest possible range in diameter for 2009 BD. For different configurations, the black lines are shifted to smaller diameters. The red and blue lines represent the two possible solutions of the orbital model in thermal inertia for the possible ranges in diameter (dashed lines illustrate $1 \sigma$ uncertainties, see text). The flux densities used in the production of this plot represent IRAC channel 2 in-band flux densities and include contributions from reflected solar light. 

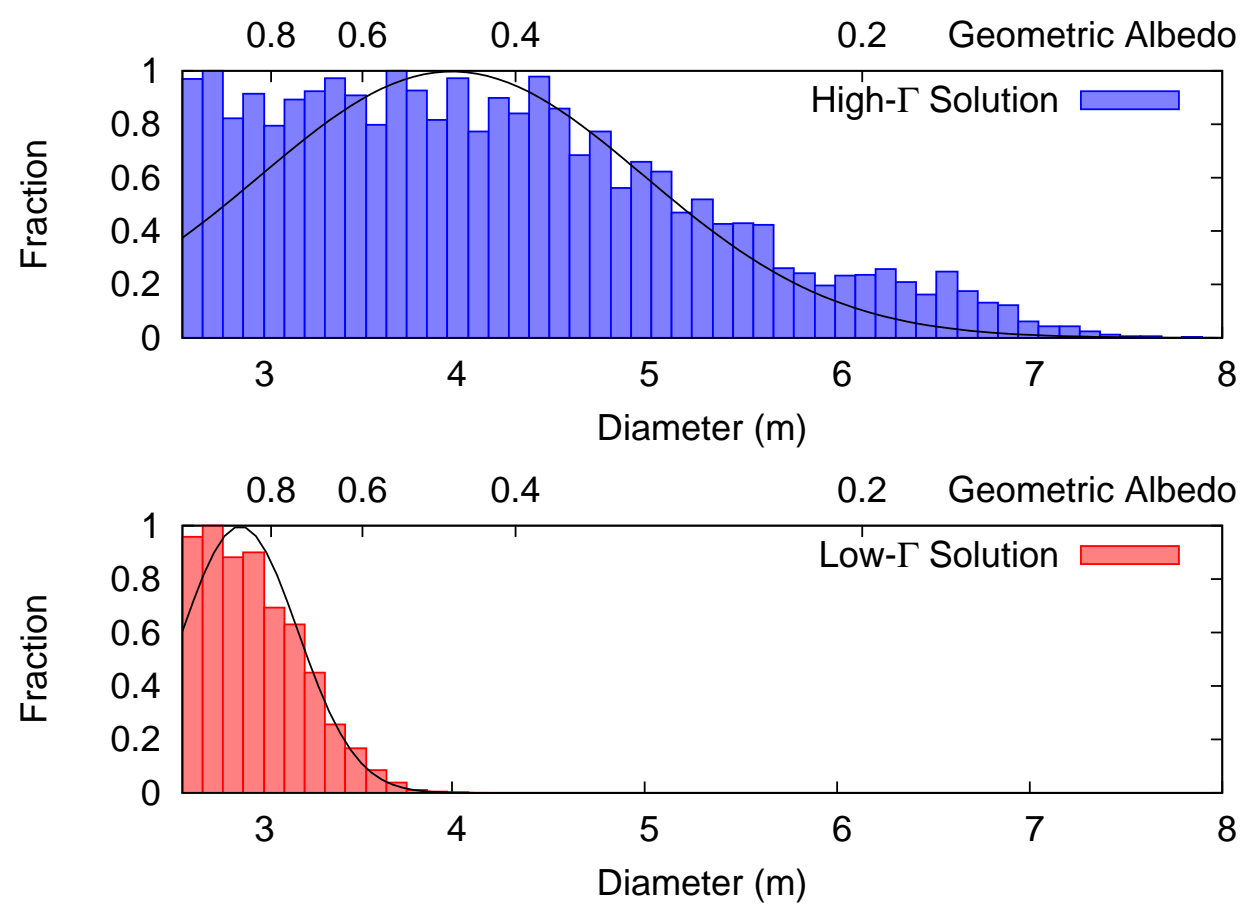

Fig. 3.- Diameter distributions of the low- $\Gamma$ (bottom) and high- $\Gamma$ (top) solutions. Overplotted black lines are normal distributions fitted to the distributions. We derive $2.9 \pm 0.3 \mathrm{~m}$ for the low- $\Gamma$ solution and $4 \pm 1 \mathrm{~m}$ for the high- $\Gamma$ solution with a lower limit of $2.6 \mathrm{~m}$ for both solutions.

Table 1. Physical Properties of 2009 BD

\begin{tabular}{rcc}
\hline \hline Parameter & Low- $\Gamma$ Solution & High- $\Gamma$ Solution \\
\hline Diameter $d(\mathrm{~m})$ & $2.9 \pm 0.3$ & $4 \pm 1$ \\
Albedo $p_{V}$ & $0.85_{-0.10}^{+0.20}$ & $0.45_{-0.15}^{+0.35}$ \\
Obliquity $\gamma\left({ }^{\circ}\right)$ & $170_{-15}^{+10}$ & $180_{-5}^{+0}$ \\
AMR $\Psi\left(\times 10^{-4} \mathrm{~m}^{2} \mathrm{~kg}^{-1}\right)$ & $1.8_{-0.2}^{+0.3}$ & $2.2_{-0.2}^{+0.4}$ \\
Bulk Density $\rho\left(\mathrm{g} \mathrm{cm}^{-3}\right)$ & $2.9_{-0.5}^{+0.5}$ & $1.7_{-0.4}^{+0.7}$ \\
Macroporosity $(\%)$ & $10_{-10}^{+20}$ & $45_{-30}^{+15}$ \\
Total Mass (Metric Tons) & $36_{-8}^{+10}$ & $55_{-25}^{+30}$ \\
Thermal Inertia $\Gamma$ (SI units) & $30_{-10}^{+20}$ & $2000 \pm 1000$ \\
\hline
\end{tabular}

Note. - Uncertainties depict the $1 \sigma$ confidence interval. 


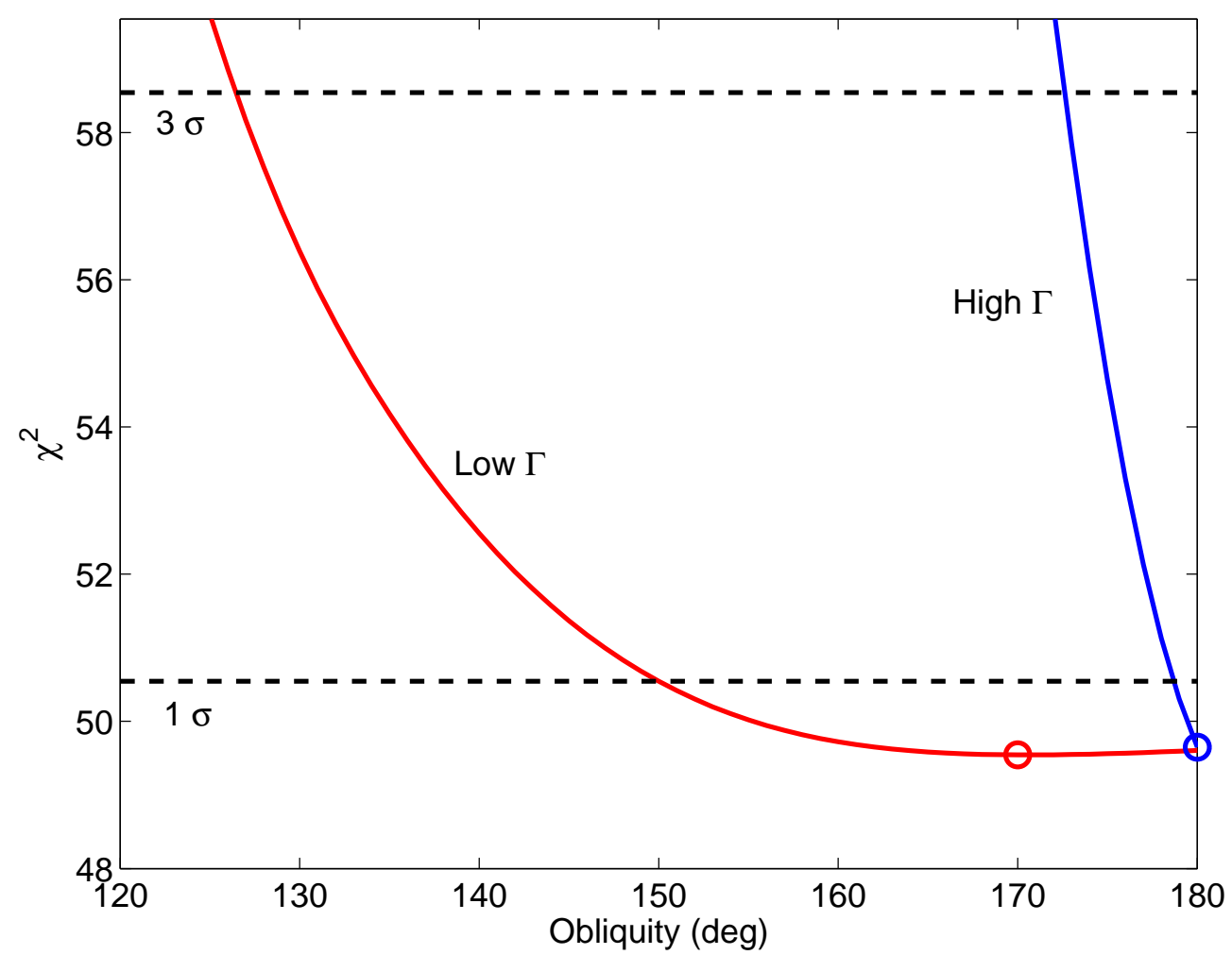

Fig. 4. - $\chi^{2}$ of the orbital fit as a function of the obliquity. The circles indicate the minima of the solutions (low and high- $\Gamma$ ). The low- $\Gamma$ solution allows for obliquities $\gamma \geq 150^{\circ}$ at the $1 \sigma$ level, $\gamma \geq 130^{\circ}$ at the $3 \sigma$ level. The high- $\Gamma$ solution allows for $\gamma>175^{\circ}$ at the $3 \sigma$ level. This plot was generated from the solution-specific diameter ranges, but looks the same for any other diameter range. 


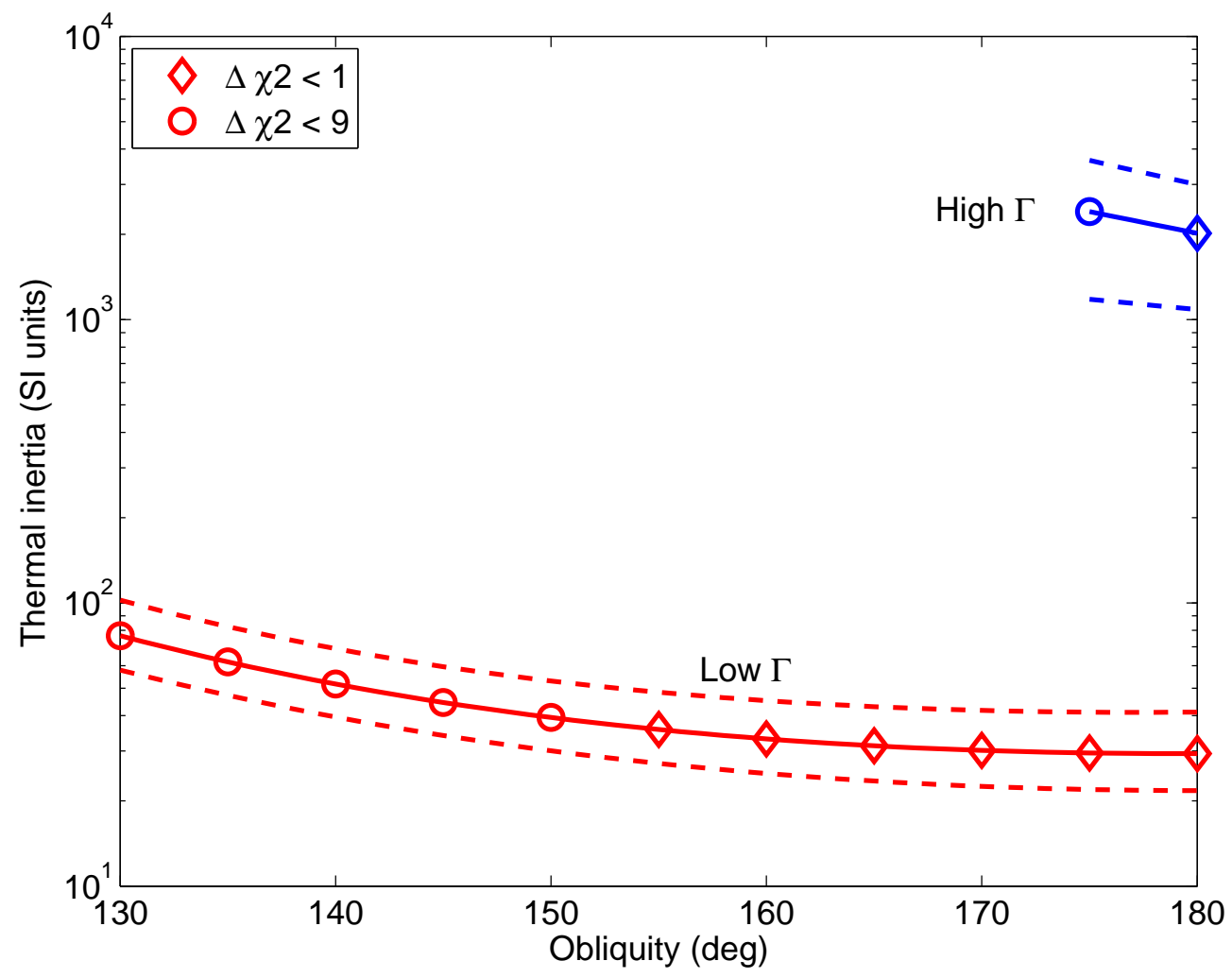

Fig. 5. - Thermal inertia as a function of the obliquity for the low- $\Gamma$ and high- $\Gamma$ solutions. Continuous lines give the most likely solution based on the numerical simulation; dashed lines indicate the $1 \sigma$ confidence interval. Symbols indicate $\Delta \chi^{2}$, the difference in $\chi^{2}$ from the respective minimum value of $\chi^{2}$. Note that $\Delta \chi^{2}<1$ (diamonds) refers to the $1 \sigma$ and $\Delta \chi^{2}<9$ (circles) to the $3 \sigma$ confidence interval in $\gamma$, as shown in Figure 4 . It is obvious from this plot that both the low- $\Gamma$ and the high- $\Gamma$ solution cover very distinct ranges in thermal inertia over the physically meaningful range of $\gamma$. 


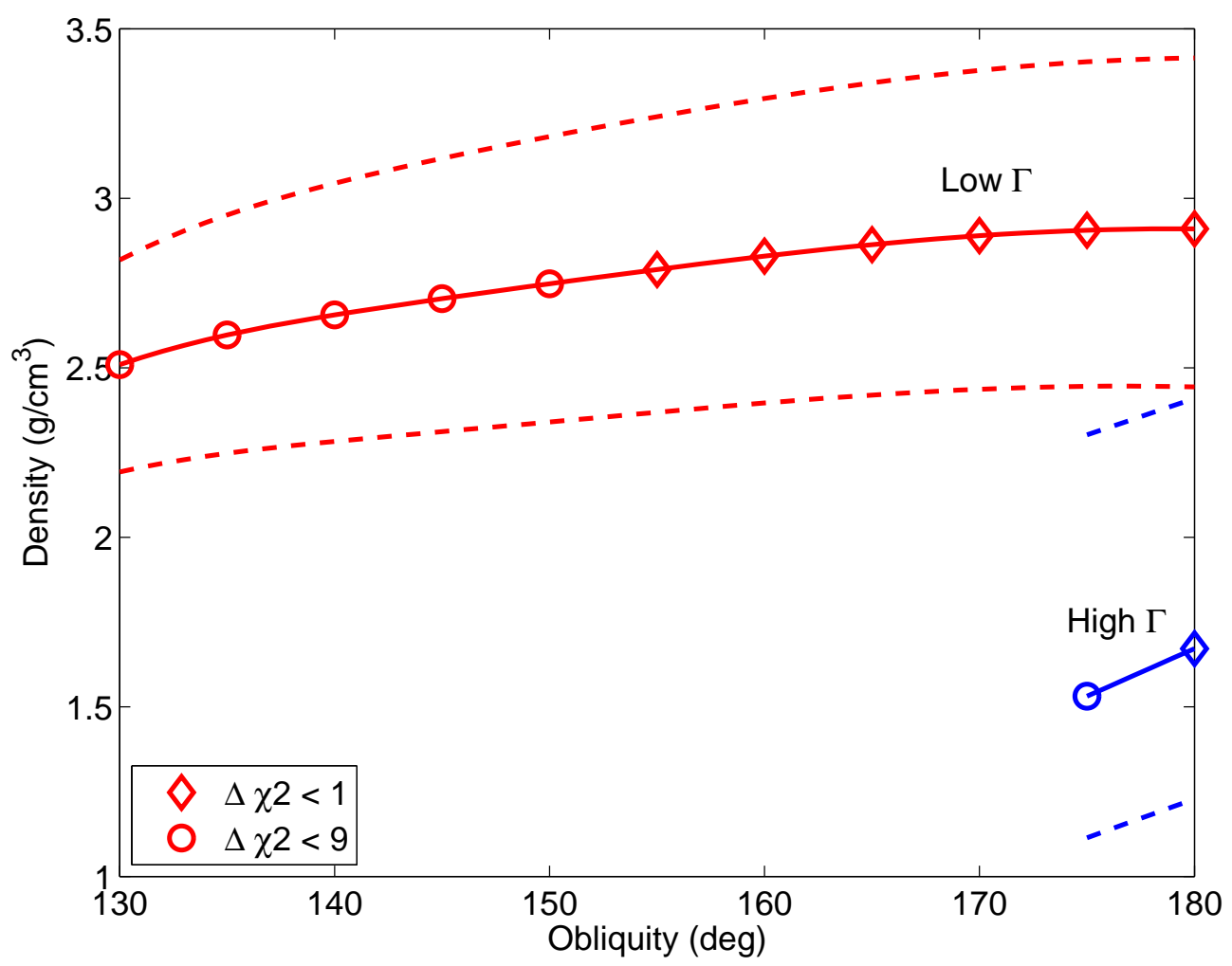

Fig. 6. - Bulk density as a function of the obliquity for the low- $\Gamma$ and high- $\Gamma$ solutions. Symbols and lines have the same meanings as in Figure 5 . 


\section{Discussion}

\subsection{Observations and Spitzer Pointing}

2009 BD was not detected in our observations, and was fainter than expected (see Figure 1). Furthermore, the final sensitivity was lower than predicted: a $5 \sigma$ detection would have been anticipated at $0.78 \mu \mathrm{Jy}$ in channel 2 , whereas we are only able to derive a $3 \sigma$ upper limit at this flux density. The lower sensitivity is possibly a result of the fact that 2009 BD moved only a short distance over the integration time, compromising the background removal and increasing the background noise of the final mosaic.

To verify that our non-detection is meaningful we have carefully checked to ensure that the Spitzer pointing was correct and the 2009 BD ephemeris predictions were accurate. To check the Spitzer pointing we have compared the most current predicted position with the position assumed by Spitzer for each frame. We find a mean discrepancy between the Spitzer pointing and the orbital predictions of $4.7 \pm 0.3^{\prime \prime}$ in right ascension and $1.6 \pm 0.2^{\prime \prime}$ in declination. This small discrepancy is readily explained by the fact that the predictions are based on a more recent Spitzer ephemeris than was available during the Spitzer observations; Spitzer position errors can be a few hundred km, leading to 3-6" pointing errors for 2009 BD. Therefore, the new position prediction is superior to the one assumed at the time of the observations. The ellipse in Figure 1 marks this updated position, which does not show a detection of 2009 BD.

So far we have shown that the searched position matches the predicted position, but to have confidence in the non-detection analysis we must also show that 2009 BD was in fact close to the predicted position. To this end we compared our nominal plane-of-sky predictions from JPL Solution 41 to the results of various alternate orbital solutions, e.g., taking into account $A 1$ only, both $A 1$ and $A 2$, gravitational effects only, and different outlier rejection schemes (Carpino et al. 2003), with the result that 2009 BD was always near the nominal prediction. We also tried to fit synthetic observations that were $\sim 5^{\prime \prime}$ from the prediction, but this consistently led to unrealistic residuals for the other observations. From this we conclude that 2009 BD could not be even $\sim 5^{\prime \prime}$ from its predicted position.

\subsection{Physical Properties}

By combining the orbital and the thermophysical model we are able to constrain a variety of physical properties of 2009 BD by taking advantage of the mutual dependencies of the individual properties. The range of each physical property is confined based on purely 
physical considerations. The only properties that are not derived as part of the modeling process are $H, G$, and $P$ (see Section 3), which are based on observations of 2009 BD or general properties of the asteroid population. We discuss those physical properties that are constrained in the modeling process.

We derive a volume-equivalent diameter of $2.9 \pm 0.3 \mathrm{~m}$ and a geometric albedo of $p_{V}=$ $0.85_{-0.10}^{+0.20}$ for the low- $\Gamma$ solution and $d=4 \pm 1 \mathrm{~m}$ and $0.45_{-0.15}^{+0.35}$ for the high- $\Gamma$ solution. Both albedo solutions exhibit relatively high albedos compared to albedo measurements of other NEOs (Trilling et al. 2010; Mainzer et al. 2011). The diameter results agree within uncertainties, allowing for a more generalized formulation: 2009 BD's diameter is $2.6<d<$ $7 \mathrm{~m}$ at a $3 \sigma$ confidence level. Note that despite the fact that we did not detect $2009 \mathrm{BD}$, we are able to constrain the diameter in both cases with uncertainties that are rather low compared to the typical 20\% diameter uncertainty derived from ExploreNEOs data (Harris et al. 2011). We are able to confine the diameter to such a narrow range due to constraints given by the physics of the orbital model and the upper-limit flux density derived from our observations. As a matter of fact, a diameter lower than $2.6 \mathrm{~m}$ implies a high Bond albedo, which in turn reduces the size of the Yarkovsky effect and prevents our model from matching the magnitude of the observed acceleration. The advantage of this definition of the lower limit is that we do not apriori rule out the possibility that 2009 BD has an extremely high albedo, which is the case for the low- $\Gamma$ solution. Although relatively rare, similarly high albedos have been found by both the ExploreNEOs (Trilling et al. 2010) and the NEOWISE projects (Mainzer et al. 2011). Based on spectral work by Thomas et al. (2011), both albedo results are compatible with a E/S/V/Q-type taxonomic classification for 2009 BD.

We compare the derived bulk density solutions for 2009 BD with measured bulk densities of asteroids with diameters of $10 \mathrm{~km}$ or smaller. Density data are taken from the list compiled by Baer et al. (2012) and the literature (see Table2). All measurements are plotted in Figure 7. Macroporosity is derived as unity minus the ratio of the asteroid's bulk density and the bulk density of meteorite equivalent material. Meteorite equivalent bulk densities are taken from Britt et al. (2002), Table 2. Due to ambiguities in the identification of the asteroidal origin of meteoritic material, we use average bulk densities derived from meteoritic material as proxies for asteroidal bulk densities of individual taxonomic types. Hence, we assume $\mathrm{S}$ and Q-type asteroids to have an average bulk density of $3.3 \pm 0.1 \mathrm{~g} \mathrm{~cm}^{-3}$, as derived from $\mathrm{H} / \mathrm{L} / \mathrm{LL}$ ordinary chondrites, and C-type and B-type asteroids to have an average bulk density of $2.6 \pm 0.5 \mathrm{~g} \mathrm{~cm}^{-3}$, as derived from different types of carbonaceous chondrites. For V-type asteroids we assume an average bulk density of $2.9_{-04}^{+0.5} \mathrm{~g} \mathrm{~cm}^{-3}$ as derived from howardite-eucrite-diogenite meteorites (Macke et al. 2011). Consolmagno et al. (2008) give a mean bulk density of enstatite chondrites, which likely originate from E-type asteroids, of $3.5 \pm 0.2 \mathrm{~g} \mathrm{~cm}^{-3}$. 
Figure7 (center panel) shows that most objects with diameters $100 \mathrm{~m}<d<10 \mathrm{~km}$ have macro-porosities higher than 30\%, consistent with a rubble-pile nature (Britt et al. 2002). There is no clear trend in either bulk density or macroporosity with the diameter of the object. Using the derived bulk density of $2009 \mathrm{BD}\left(2.9_{-0.5}^{+0.5} \mathrm{~g} \mathrm{~cm}^{-3}\right.$ for the low- $\Gamma$ solution, $1.7_{-0.4}^{+0.7} \mathrm{~g} \mathrm{~cm}^{-3}$ for the high- $\Gamma$ solution), and a mean bulk density of $(3.2 \pm 0.3) \mathrm{g} \mathrm{cm}^{-3}$ derived as the average of the $\mathrm{S} / \mathrm{Q} / \mathrm{V} / \mathrm{E}$-type asteroid material bulk densities listed above, we derive a degree of macroporosity of $10_{-10}^{+20 \%}$ or $45_{-30}^{+15 \%}$. Hence, our low- $\Gamma$ solution is most consistent with a monolithic nature of $2009 \mathrm{BD}$, whereas the high- $\Gamma$ solution suggests a rubble-pile nature (Britt et al. 2002).

Previous measurements of the thermal inertia of asteroids revealed values in the range 10-100 SI units for large main belt asteroids and higher values up to 1000 SI units for NEOs (see, e.g., Delbo' et al. 2007, and references therein). The lower thermal inertia of large bodies is generally ascribed to the presence of a thick layer of regolith, which has a low thermal inertia (see Section 1, as well as Spencer et al. 1989; Putzig et al. 2005). The righthand panel of Figure 7 plots thermal inertia measurements of asteroids with $\sim 0.1<d<$ $10 \mathrm{~km}$. The plot reveals that 2009 BD's thermal inertia is extreme in this range, irrespective of which of our solutions better describes reality. The low- $\Gamma$ solution is consistent with the presence of regolith, whereas the high- $\Gamma$ solution is consistent with a bare-rock nature of 2009 BD. A slight trend of increasing thermal inertia with decreasing diameter is visible in Figure 7, which was already discussed by Delbo' et al. (2007). Our high- $\Gamma$ solution seems to be more consistent with this trend, presuming that this trend is valid for asteroids in the size regime of $2009 \mathrm{BD}$.

We summarize the properties of 2009 BD created by our two solutions in Figure 8 . The low- $\Gamma$ solution suggests that $2009 \mathrm{BD}$ is a $\sim 3 \mathrm{~m}$ sized rocky body $\left(\rho=2.9 \mathrm{~g} \mathrm{~cm}^{-3}\right)$ that is covered with a physically thin but optically thick layer of regolith-like material, causing it to exhibit a low thermal inertia ( $\Gamma=30$ SI units) and a high bulk density. This picture seems realistic in the sense that models show that even small asteroids can retain a layer of fine-grained dust on their surfaces (Scheeres et al. 2010; Sánchez \& Scheeres 2013). The picture created by the high- $\Gamma$ solutions shows 2009 BD as a 4 m-sized rubble-pile asteroid $\left(\rho=1.7 \mathrm{~g} \mathrm{~cm}^{-3}\right)$ that consists of individual bare rock slabs ( $\Gamma=2000$ SI units) and exhibits a macroporosity of $45 \%$. This scenario seems to be more realistic due to the lower albedo that is required for this configuration. Despite the fact that some properties seem to favor one of the two results at a time, we are unable to rule out either of the configurations based on physical reasoning. In order to be able to commit to either solution, additional observations are necessary that are able to pinpoint one decisive physical property of 2009 BD, e.g., its thermal inertia or its bulk density. The thermal inertia can be further constrained using additional infrared observations or using in-situ measurements. The bulk density can be 
Table 2. Physical Properties of Small Asteroids

\begin{tabular}{|c|c|c|c|c|c|c|c|}
\hline Object & $\begin{array}{l}\text { Diameter } \\
(\mathrm{km})\end{array}$ & Albedo & $\begin{array}{l}\text { Tax. } \\
\text { Type }\end{array}$ & $\begin{array}{l}\text { Bulk Density } \\
\quad\left(\mathrm{g} \mathrm{cm}^{-3}\right)\end{array}$ & $\begin{array}{l}\text { MPor. } \\
(\%)\end{array}$ & $\begin{array}{c}\Gamma \\
(\mathrm{SI})\end{array}$ & Ref. \\
\hline (1580) Betulia & $4.57 \pm 0.46$ & $0.08 \pm 0.02$ & $\mathrm{C}$ & $\ldots$ & $\ldots$ & $180 \pm 50$ & 1 \\
\hline (1862) Apollo & $1.55 \pm 0.07$ & $0.20 \pm 0.02$ & $\mathrm{Q}$ & $2.85 \pm 0.68$ & $15_{-15}^{+25}$ & $140_{-100}^{+140}$ & 2,3 \\
\hline (3749) Balam & $(7.2 \pm 0.4)^{\mathrm{a}}$ & $(0.15)^{\mathrm{a}}$ & $(\mathrm{S})^{\mathrm{b}}$ & $(2.61 \pm 0.45)^{\mathrm{a}}$ & $20_{-15}^{+15}$ & $\ldots$ & 4 \\
\hline (3908) Nyx & $1.0 \pm 0.2$ & $0.15 \pm 0.08$ & $\mathrm{~V}$ & $0.9 \pm 0.2^{\mathrm{c}}$ & $70_{-15}^{+10}$ & $\ldots$ & $5,6,7$ \\
\hline (25143) Itokawa & $0.320 \pm 0.001$ & $0.30 \pm 0.10$ & $\mathrm{~S}$ & $1.90 \pm 0.13$ & $40_{-10}^{+10}$ & $700 \pm 100$ & $1,8,9$ \\
\hline (33342) 1998 WT24 & $0.35 \pm 0.04$ & $0.6 \pm 0.2$ & $\mathrm{E}$ & $\ldots$ & $\ldots$ & $200 \pm 100$ & 1,10 \\
\hline (54509) YORP & $0.09 \pm 0.01$ & $0.20 \pm 0.02$ & $\mathrm{~S} / \mathrm{V}$ & $\ldots$ & $\ldots$ & $700 \pm 500$ & 1 \\
\hline (66391) $1999 \mathrm{KW} 4$ & $1.33 \pm 0.07$ & $(0.25)^{\mathrm{d}}$ & $\mathrm{S}$ & $2.00 \pm 0.26$ & $40_{-10}^{+10}$ & $\ldots$ & 5,11 \\
\hline (101955) Bennu & $0.50 \pm 0.02$ & $0.05 \pm 0.01$ & $\mathrm{~B}$ & $1.2 \pm 0.1$ & $55_{-15}^{+10}$ & $650 \pm 100$ & 12,13 \\
\hline (162173) 1999 JU3 & $0.87 \pm 0.03$ & $0.07 \pm 0.01$ & $\mathrm{C}$ & $\ldots$ & -15 & $400 \pm 200$ & 3,14 \\
\hline (175706) 1996 FG3 & $1.71 \pm 0.07$ & $0.044 \pm 0.004$ & $\mathrm{C}$ & $\ldots$ & $\ldots$ & $120 \pm 50$ & 15,16 \\
\hline (185851) 2000 DP107 & $0.81 \pm 0.18$ & $(0.14)^{\mathrm{d}}$ & $(S)^{d}$ & $1.65 \pm 0.84$ & $50_{-30}^{+25}$ & $\ldots$ & 17 \\
\hline (308635) 2005 YU55 & $0.31 \pm 0.01$ & $0.07 \pm 0.01$ & $\mathrm{C}$ & $\ldots$ & $\ldots$ & $580 \pm 230$ & 18,19 \\
\hline (341843) 2008 EV5 & $0.37 \pm 0.01$ & $0.13 \pm 0.05$ & $\mathrm{C}$ & $\ldots$ & $\ldots$ & $450 \pm 60$ & 19,20 \\
\hline 2000 UG11 & $0.23 \pm 0.03$ & $(0.23)^{\mathrm{d}}$ & $(\mathrm{S})^{\mathrm{d}}$ & $1.47 \pm 0.7$ & $55_{-25}^{+20}$ & $\ldots$ & 21 \\
\hline $2002 \mathrm{CE} 26$ & $3.50 \pm 0.40$ & 0.07 & $(\mathrm{C})^{\mathrm{e}}$ & $0.9 \pm 0.5$ & $65_{-30}^{+20}$ & $\ldots$ & 22 \\
\hline 2002 NY40 & $0.28 \pm 0.03$ & $0.34 \pm 0.06$ & $\mathrm{Q}$ & $\ldots$ & -30 & $550 \pm 450$ & 23,24 \\
\hline $2003 \mathrm{YT} 1$ & $1.06 \pm 0.06$ & $(0.52)^{\mathrm{d}}$ & $\mathrm{V}$ & $2.01 \pm 0.70$ & $30_{-30}^{+30}$ & $\ldots$ & 25,26 \\
\hline
\end{tabular}

Note. - This table lists measured physical properties of asteroids with diameters of $10 \mathrm{~km}$ or smaller. Data in brackets are based on assumptions (see below). In the case of multi-component systems, diameters and bulk densities refer to the average numbers of the combined system (diameters are those of a volume-equivalent sphere). The macroporosity ("MPor.") is derived through division of the asteroid's bulk density by the bulk density of the respective meteorite equivalent material (see text for details). Comparison data for 2009 BD can be found in Table 1

abased on an assumed albedo

b based on its Flora family membership (Marchis et al. 2008)

'density estimate assumes a thermal inertia according to Delbo' et al. (2007)

dalbedo derived from the equivalent diameter and the $H$ magnitude (JPL Small-Body Database Browser 2013)

etaxonomic type assigned based on albedo determinations by Thomas et al. (2011)

References. - (1) Mueller (2007); (2) Rozitis et al. (2013); (3) Bus \& Binzel 2002); (4) Marchis et al. (2008); (5) Binzel et al. (2004); (6) Benner et al. (2002); (7) Farnocchia et al. (2014b); (8) Fujiwara et al. (2006); (9) Abe et al. (2006); (10) Kiselev et al. (2002); (11) Ostro et al. (2006); (12) Müller et al. (2012); (13) Cheslev et al. (2014); (14) Müller et al. (2011); (15) Wolters et al. (2011); (16) Thomas et al. (2011); (17) Margot et al. (2002); (18) Müller et al. (2013); (19) Somers et al. (2010); (20) Alí-Lagoa et al. (2014); (21) Margot et al. (2002b); (22) Shepard et al. (2006); (23) Roberts et al. (2007); (24) Müller et al. (2004); (25) Brooks (2006); (26) Sanchez et al. (2013). 

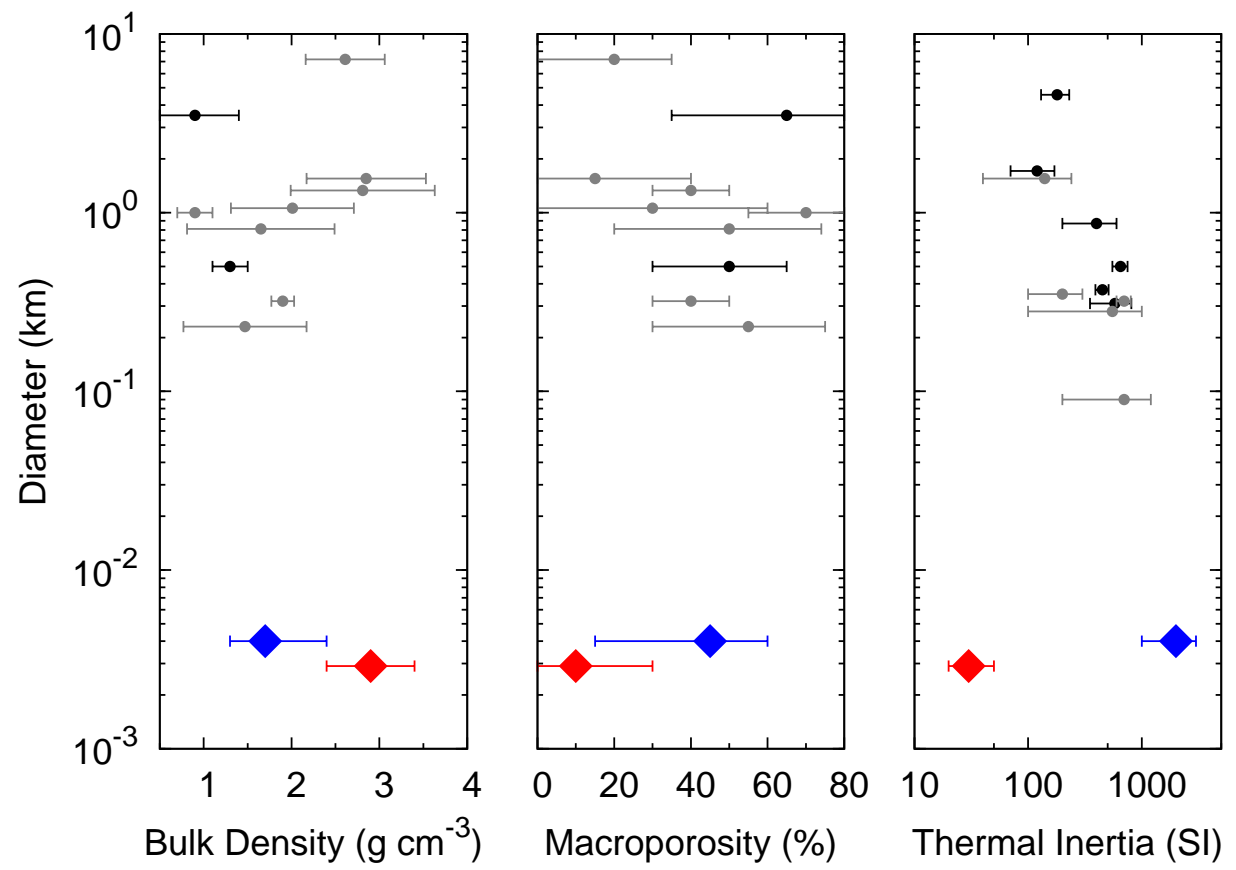

Fig. 7.- Physical properties of known asteroids with diameters of $10 \mathrm{~km}$ or less, as a function of their diameter: bulk density (left), macroporosity (center), and thermal inertia (right). Grey circles depict stony asteroid types (S/Q/V/E), black circles carbonaceous types (B/C) (according to Table 2). The low- $\Gamma$ solution of 2009 BD is indicated as a red diamond, the high- $\Gamma$ solution as a blue diamond in each plot. A large degree of variety in bulk densities and macroporosity is obvious, and there is no clear trend between either of them and diameter. Thermal inertia exhibits a slight trend of smaller objects having higher thermal inertia (see Delbo' et al. 2007). Data plotted here are tabulated in Tables 1 and 2 . 
independently derived by measuring the gravitational attraction of the object on a nearby body or a rendezvous spacecraft.

\subsection{Discussion of the Modeling Technique}

The probabilistic approach taken in this work to constrain the physical properties of $2009 \mathrm{BD}$ is unique. We have to acknowledge that a full validation of the methods presented in this paper is not yet possible. 2009 BD is currently the only asteroid for which both the Yarkovsky and solar radiation pressure forces can be measured from astrometric observations, which are used here to constrain the object's bulk density and thermal inertia. An independent validation of our modeling approach would require an asteroid with non-gravitational perturbations and physical model independently characterized. The first object for which such a wealth of data is anticipated will be NEO (101955) Bennu, the OSIRIS-REx mission target.

Instead, we note that both the thermophysical and the dynamical models are individually well-tested. The thermophysical model used in this work is based on and has been extensively tested against the model discussed by Mueller (2007), which was applied in a number of publications (e.g., Harris et al. 2007; Mueller et al. 2010). The model of the Yarkovsky forces is based on work done by Vokrouhlický et al. (2000), which is used to describe the Yarkovsky effect observed in a number of objects (e.g., Cheslev et al. 2003; Vokrouhlickv́ et al. 2008; Farnocchia et al. 2013; Chesley et al. 2014; Farnocchia and Cheslev 2014; Farnocchia et al. 2014b). Also, the solar radiation pressure model (Vokrouhlickv́ \& Milani 2000) was used to refine orbits of small asteroids (e.g., Micheli et al. 2012, b, 2013). Note that in all previous works in which either model has been used, the resulting physical properties are within reasonable ranges.

Both the orbital and the thermophysical model assume a spherical shape of 2009 BD. Emery et al. (2014) have shown that using the real shape of (101955) Bennu, instead of assuming a spherical shape, lowers the thermal inertia of that object by a factor of 2 . The case of Bennu shows that shape information can impact the physical parameter results. We investigate the possible impact of an irregular shape on our results. 2009 BD has a rotation period $P \geq 3 \mathrm{hrs}$ (Tholen et al. 2013) with a lightcurve magnitude of $\geq 0.25 \mathrm{mag}$ (B. Ryan, private communication 2014), which suggests an elongation $b / a \leq 0.8$ for a triaxial ellipsoid with relative dimensions $(a, b, c)$. Assuming a rotation period $3<P \ll 25$ hrs, any elongation effects are averaged out during our $25 \mathrm{hrs}$ integration, leading to physical properties of a volume-equivalent sphere. We further investigate the effect of a possible flattening of $2009 \mathrm{BD}$, by assuming the shape of a triaxial ellipsoid with axes $b / a=0.7$ and 
$c / b=0.7$, which is quite typical among larger asteroids. We find that the smaller crosssection of the triaxial shape compared to that of a spherical shape requires a reduction of the bulk density of $15-20 \%$ to provide the observed magnitude of the solar radiation pressure force. A numerical simulation of the Yarkovsky forces (Vokrouhlický et al. 2000) suggests that the thermal inertia estimates might be lower by as much as a factor of 2 , which has also

been found for Bennu by Emery et al. (2014). The smaller thermal inertia in turn reduces the diameters found for both solutions (see Figures 2 and 3). Note that the changes to the individual physical properties found as part of this simulation are mostly within the uncertainties derived assuming a spherical shape. Since there is no certainty on the shape of 2009 BD we stick to the results based on a simple spherical shape, which are still valid within the uncertainties, assuming a triaxial shape. Additional information on the shape of 2009 BD might require a re-assessment of our results in the future.

\subsection{Implications}

Our results show that the volume-equivalent diameter of $2009 \mathrm{BD}, 2.6<d<7.0 \mathrm{~m}(3 \sigma)$, is most likely smaller than the size range aimed for in the ARRM mission design (7-10 m, see above). However, its total mass is roughly $1 / 10$ of the mass aimed for in the current design, reducing efforts necessary to alter the orbit of the asteroid. The final decision on 2009 BD's suitability as a mission target is beyond the scope of this work, but a potential mission to $2009 \mathrm{BD}$ will be able to resolve the solution degeneracy we found for the physical properties of this object.

The two scenarios based on our data and presented in Section 5.2 show 2009 BD either as a rocky object covered with regolith-like material or a loose conglomerate of bare rocks. Either scenario reveals this object as rather exotic compared to other known asteroids. Hence, 2009 BD may not belong to the normal population of NEOs that have their origins in the main belt, accounting for its very Earth-like orbit. It has been suggested that ejecta from impacts on the Moon could end up in Earth-like orbits; another possibility is that $2009 \mathrm{BD}$ is a man-made object (Micheli et al. 2012). A value for $p_{V}$ of around 0.45 or 0.85 is much higher than the Moon's albedo albedo of 0.11 (de Pater \& Lissauer 2001), which would appear to reduce the likelihood that 2009 BD has a lunar origin. A section of a spent rocket booster would have a high albedo, but the densities derived here (for both the low and the high- $\Gamma$ solutions) are far higher than values associated with, for example, hollow rocket fuel tanks. Specifically, the values of the area-to-mass ratio $(\Psi)$ listed in Table 1 , which are substantially independent of the diameter estimate, are 1-2 orders of magnitude less than that of artificial objects. The available astrometry contradicts area-to-mass ratios 


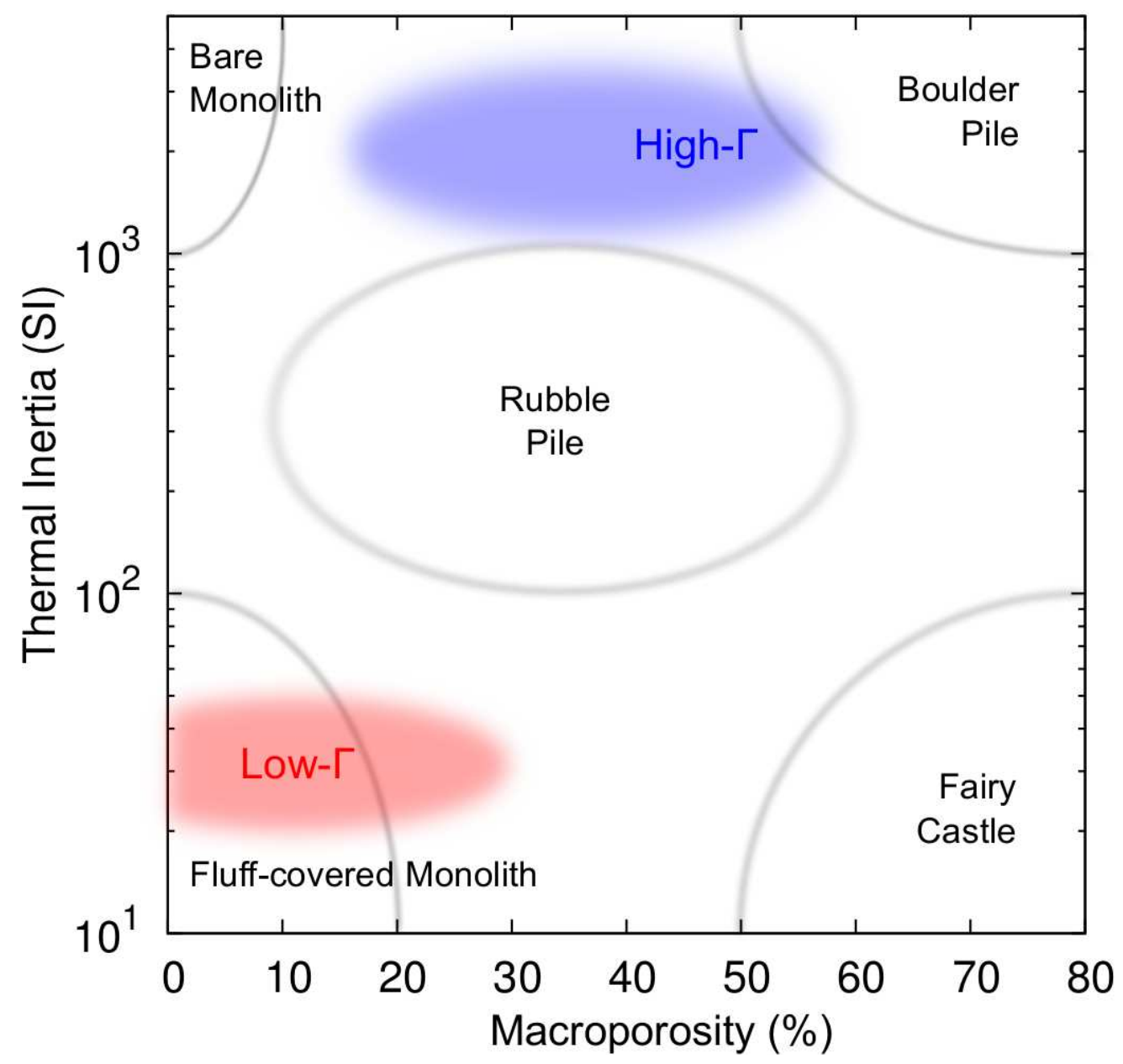

Fig. 8.- Schematic diagram of the nature of 2009 BD. Grey lines indicate the different possible configurations as a function of thermal inertia and macroporosity. The red and the blue cloud symbolize the $1 \sigma$ confidence intervals of the low and high- $\Gamma$ solutions, respectively. Note that the border lines of the different configurations are not as strict as shown here. 
compatible with a spent booster.

While our results do not appear to favor any of the more exotic origins for 2009 BD suggested by its very Earth-like orbit, they emphasize the puzzling nature of this object and the need for further observations of this and similar objects.

\section{Summary}

We derive two physically possible solutions for the physical properties of 2009 BD from our Spitzer observations, using thermophysical modeling and modeling of the nongravitational forces acting upon this body. The first solution shows 2009 BD as a $2.9 \pm 0.3 \mathrm{~m}$ sized massive rock body $\left(\rho=2.9 \pm 0.5 \mathrm{~g} \mathrm{~cm}^{-3}\right)$ with an extremely high albedo of $p_{V}=$ $0.85_{-0.10}^{+0.20}$ that is covered with regolith-like material, causing it to exhibit a low thermal inertia $\left(\Gamma=30_{-10}^{+20}\right.$ SI units). The second solution suggests $2009 \mathrm{BD}$ to be a $4 \pm 1 \mathrm{~m}$-sized rubble-pile asteroid $\left(\rho=1.7_{-0.4}^{+0.7} \mathrm{~g} \mathrm{~cm}^{-3}\right.$ ) with an albedo of $0.45_{-0.15}^{+0.35}$ that consists of individual bare rock slabs $(\Gamma=2000 \pm 1000$ SI units). We are unable to rule out either solution with the current knowledge.

The authors of this work thank Tom Soifer, Director of the Spitzer Space Telescope, for the time allocation to observe 2009 BD. We also would like to thank Paul Chodas for his support and many informative conversations. We thank an anonymous referee for useful suggestions that improved this manuscript. D. Farnocchia was supported for this research by an appointment to the NASA Postdoctoral Program at the Jet Propulsion Laboratory, California Institute of Technology, administered by Oak Ridge Associated Universities through a contract with NASA. The work of S. Chesley was conducted at the Jet Propulsion Laboratory, California Institute of Technology under a contract with the National Aeronautics and Space Administration. The work of D. Vokrouhlický was partially supported by the Grant Agency of the Czech Republic (grant P209-13-01308S). J. L. Hora and H. A. Smith acknowledge partial support from Jet Propulsion Laboratory RSA \#1367413. This work is based on observations made with the Spitzer Space Telescope, which is operated by the Jet Propulsion Laboratory, California Institute of Technology under a contract with NASA.

Facilities: Spitzer.

\section{REFERENCES}

Abe, M., Takagi, Y., Kitazato, K., Abe, S., Hiroi, T. et al. 2006, Science, 312, 1334 
Alí-Lagoa, V., Lionni, L., Delbo', M., Gundlach, B., Blum, J. et al. 2014, eprint arXiv:1310.6715

Baer, J., Chesley, S., and Britt, D. 2012, eds., Asteroid Masses V3.0. EARA-COMPIL-5-ASTMASS-V3.0. NASA Planetary Data System, URL: http://sbn.psi.edu/pds/resource/astmass.html

Benner, L. A. M., Ostro, S. J., Hudson, R. S., Rosema, K. D., Jurgens, R. F. 2002, Icarus, 158,379

Binzel, R. P., Rivkin, A. S., Stuart, J. S., Harris, A. W., Bus, S. J. et al. 2004, Icarus, 170, 259

Bottke, W. F., Vokrouhlický, D., Rubincam, D. P., Nesvorný, D. 2006, Annual Review of Earth and Planetary Sciences, 34, 157

Britt, D. T., Yeomans, D., Housen, K., Consolmagno, G. 2002, in Asteroids III, W. F. Bottke Jr., A. Cellino, P. Paolicchi, and R. P. Binzel (eds), University of Arizona Press, Tucson, 485

Brooks, H. E. 2006, BAAS, 38, 934

Bus, S. J., Binzel, R. P. 2002, Icarus, 158, 146

Buzzi, L., Hormuth, F., Bittesini, L., Fabris, F., Pettarin, E. et al. 2009, MPEC 2009-B14

Carpino, M., Milani, A., Chesley, S. R. 2003, Icaurs, 166, 248

Chapman, C. R. 1978, in Asteroids: An Exploration Assessment, D. Morrison and W. C. Wells (eds.), NASA Conf. Publ. 2053, 145

Chesley, S. R., Ostro, S. J., Vokrouhlický, D., Čapek, D., Giorgini, J. D. et al. 2003, Science, 302,1739

Chesley, S. R., Farnocchia, D., Nolan, M. C. et al. 2014, submitted to Icarus, arXiv:1402.5573

Consolmagno, G., Britt, D., Macke, R. 2008, Chemie der Erde, 68, 1

Delbo', M., dell'Oro, A., Harris, A. W., Mottola, S., \& Mueller, M. 2007, Icarus, 190, 236

de Pater, I. \& Lissauer, J. 2001, Planetary Sciences, Cambridge University Press

Emery, J. P., Ferndandez, Y. R., Kelley, M. S. P., Hergenrother, C. W., Lauretta, D. S. et al. 2014, Icarus, 234, 17 
Farnocchia, D., Chesley, S. R., Vokrouhlický, D., et al. 2013, Icarus, 224, 1

Farnocchia, D. and Chesley, S. R. 2014, Icarus, 229, 321

Farnocchia, D., Chesley, S. R., Tholen, D. J., Micheli, M. 2014, Celestial Mechanics and Dynamical Astronomy, DOI 10.1007/s10569-014-9536-9

Fazio, G. G., Hora, J. L., Allen, L. E., Ashby, M. L. N., Barmby, P. et al. 2004, ApJS, 154, 10

Fujiwara, A., Kawaguchi, J., Yeomans, D. K., Abe, M., Mukai, T. et al. 2006, Science, 312, 1330

Harris, A. W. 1998, Icarus, 131, 291

Harris, A. W., Mueller, M., Delbó, M., Bus, S. J. 2007, Icarus, 188, 414

Harris, A. W., Mommert, M., Hora, J. L., Mueller, M., Trilling, D. E. et al. 2011, AJ, 141, 75

Jakosky, B.M. 1986, Icarus, 66, 117

JPL Small-Body Database Browser, http://ssd.jpl.nasa.gov/sbdb.cgi\#top, accessed October 10,2013

JPL Small-Body Database Search Engine, http://ssd.jpl.nasa.gov/sbdb_query.cgi, accessed October 28, 2013

Kiselev, N. N., Rosenbush, V. K., Jockers, K., Velichko, F. P., Shakhovskoj, N. M et al. 2002, in Proceedings of Asteroids, Comets, Meteors - ACM 2002, ed. Barbara Warmbein. ESA SP-500. Noordwijk, Netherlands: ESA Publications Division, 887

Macke, R. J., Consolmagno, G. J., Britt, D. T. 2011, M\&PS, 46, 1842

Mainzer, A., Grav, T., Bauer, J., Masiero, J., McMillan, R. S. et al. 2011, ApJ, 743, 156

Mainzer, A., Bauer, J.,Grav, T., Masiero, J., Cutri, R. M. et al. 2014, ApJ, 784, 110

Marchis, F., Descamps, P., Berthier, J., Hestroffer, D., Vachier, F. et al. 2008, Icarus, 195, 295

Margot, J. L., Nolan, M. C., Benner, L. A. M., Ostro, S. J., Jurgens, R. F. et al. 2002, Science, 296, 1445 
Margot, J. L., Nolan, M. C., Benner, L. A. M., Ostro, S. J., Jurgens, R. F. et al. 2002b, 33rd Annual Lunar and Planetary Science Conference, abstract no.1849

Marsden, B. G., Sekanina, Z., \& Yeomans, D. K. 1973, AJ, 78, 211

Micheli, M., Tholen, D. J. \& Elliott, G. 2012, New Astronomy, 17, 446

Micheli, M., Tholen, D. J., Elliott, G. T. 2012, ACM 2012 proceedings, 1667

Micheli, M., Tholen, D. J., Elliott, G. T. 2013, Icarus, 226, 251

Mueller, M. 2007, PhD Thesis, Freie Universität Berlin

Mueller, M., Marchis, F., Emery, J. P., Harris, A. W., Mottola, S, et al., Icarus, 205, 505

Mueller, M., Delbo', M., Hora, J. L., Trilling, D. E., Bhattacharya, B. et al. 2011, AJ, 141, 109

Müller, T. G., Sterzik, M. F., Schütz, O., Pravec, P., Siebenmorgen, R. 2004, A\&A, 424, 1075

Müller, T. G., Ďurech, J., Hasegawa, S., Abe, M., Kawakami, K. et al. 2011, A\&A, 525, A145

Müller, T. G., O’Rourke, L., Barucci, A. M., Pál, A., Kiss, C. et al. 2012, A\&A, 548, 36

Müller, T. G., Miyata, T., Kiss, C., Gurwell, M. A., Hasegawa, S. et al. 2013, A\&A, 558, A97

NASA Asteroid Initiative Website, http://www.nasa.gov/mission_pages/asteroids/initiative/index.html, accessed October 24, 2013

NASA Solar System Exploration Mission Website http://solarsystem.nasa.gov/missions/profile.cfm?MCod accessed October 24, 2013

Ostro, S. J., Margot, J.-L., Benner, L. A. M., Giorgini, J. D., Scheeres, D. J. et al. 2006, Science, 314,1276

Pravec, P., Harris, A. W., Michałowski, T. in Asteroids III, W. F. Bottke Jr., A. Cellino, P. Paolicchi, and R. P. Binzel (eds), University of Arizona Press, Tucson, 113

Putzig, N. E., Mellon, M. T., Kretke, K. A., Arvidson, R. E. 2005, Icarus, 173, 325 
Richardson, D. C., Leinhardt, Z. M., Melosh, H. J., Bottke, W. F., Jr., Asphaug, E. in Asteroids III, W. F. Bottke Jr., A. Cellino, P. Paolicchi, and R. P. Binzel (eds), University of Arizona Press, Tucson, 501

Roberts, L. C., Hall, D. T., Lambert, J. V., Africano, J. L., Knox, K. T. et al. 2007, Icarus, 192, 469

Rozitis, B., Duddy, S. R., Green, S. F., Lowry, S. C. 2013, A\&A, 555, A20

Sanchez, J. A., Michelsen, R., Reddy, V., Nathues, A. 2013, Icarus, 225, 131

Sánchez, P., Scheeres, D. J. 2013, submitted, arXiv:1306.1622

Scheeres, D. J., Hartzell, C. M., Sanchez, P., Swift, M. 2010, Icarus, 2010, 968

Shepard, M. K., Margot, J.-L., Magri, C., Nolan, M. C., Schlieder, J. et al. 2006, Icarus, 184,198

Somers, J. M., Hicks, M., Lawrence, K., Rhoades, H., Mayes, D. et al. 2010, Bulletin of the American Astronomical Society, 42, 1055

Spencer, J. R., Lebofsky, L. A., Sykes, M. V. 1989, Icarus, 78, 337

Tholen, D. J., Micheli, M., Bauer, J., Mainzer, A. 2013, American Astronomical Society, DPS meeting \#45, \#101.08

Thomas, C. A., Trilling, D. E., Emery, J. P., Mueller, M., Hora, J. L. et al. 2011, AJ, 142, 85

Trilling, D. E., Mueller, M., Hora, J. L., Harris, A. W., Bhattacharya, B. et al. 2010, AJ, 140,770

Trilling, D. E., Mommert M., Mueller, M., Spahr, T. B. Hora, J. L. et al. 2013, submitted to AJ

Vokrouhlický, D. 1998, A\&A 335, 1093

Vokrouhlický, D. and Farinella, P. 1999, AJ, 118, 3049

Vokrouhlický, D., \& Milani, A. 2000, A\&A, 362, 746

Vokrouhlický, D., Milani, A., Chesley, S. R. 2000, Icarus, 148, 118

Vokrouhlický, D., Chesley, S. R., Matson, R. D. 2008, AJ, 135, 2336 
Warm Spitzer Observer's Manual, Spitzer Science Team 2012, Version 11.1, http://ssc.spitzer.caltech.edu/warmmission/propkit/som/som11.1.pdf,

Werner, M. W., Roellig, T. L., Low, F. J., Rieke, G. H., Rieke, M. et al. 2004, ApJS, 154, 1

Wolters, S. D., Rozitis, B., Duddy, S. R., Lowry, S. C., Green, S. F. et al. 2011, MNRAS, 418, 1246

Wright, E. L., Eisenhardt, P. R. M., Mainzer, A. K., Ressler, M. E., Cutri, R. M. et al., 2010, AJ, 140, 1868 